\title{
25 Research Suare \\ Experimental infection with the hookworm, Necator americanus, promotes gut microbial diversity in human volunteers with relapsing multiple sclerosis
}

Timothy Jenkins

Danmarks Tekniske Universitet

David Pritchard

University of Nottingham

Radu Tanasescu

University of Nottingham

Gary Telford

University of Nottingham

Marina Paraiakovou

National Museum of Natural History

Cris Constantinescu

University of Nottingham

Cinzia Cantacessi ( $\nabla$ cc779@cam.ac.uk)

University of Cambridge https://orcid.org/0000-0001-6863-2950

Research

Keywords: gut microbiota, Multiple sclerosis, Necator americanus

Posted Date: February 25th, 2020

DOI: https://doi.org/10.21203/rs.2.24428/v1

License: (9) (i) This work is licensed under a Creative Commons Attribution 4.0 International License.

Read Full License 


\section{Abstract}

Background Helminth-associated changes in gut microbiota composition have been hypothesised to contribute to the immune-suppressive properties of parasitic worms. Multiple sclerosis is an immunemediated autoimmune disease of the central nervous system whose pathophysiology has been recently linked to alterations of gut microbial communities.

Results In the present study we investigated, for the first time, qualitative and quantitative changes in gut microbial composition of human volunteers with remitting multiple sclerosis (RMS) prior to and following experimental infection with the human hookworm, Necator americanus $(\mathrm{N}+)$, and following anthelmintic treatment, and compared the findings with data obtained from a cohort of RMS patients subjected to placebo treatment ( PBO ). Bacterial 16S rRNA high-throughput sequencing data revealed significantly decreased microbial alpha diversity in the gut microbiota of PBO compared to $\mathrm{N}+$ subjects over the course of the trial; additionally, we observed significant differences in the abundances of several bacterial taxa with putative immune-modulatory functions between study cohorts. Parabacteroides were significantly expanded in the gut microbiota of $\mathrm{N}+$ individuals for which no relapses were recorded at the end of the trial.

Conclusions Overall, these data lend support to the hypothesis of a contributory role of parasiteassociated alterations in gut microbial composition to the immunomodulatory properties of hookworm parasites.

\section{Background}

A growing body of evidence supports a key role of infections by gastrointestinal (GI) helminth parasites in shaping the composition and function of the human gut microbiota, with significant implications for local and systemic host immunity, and metabolic potential [reviewed by 1]. Notably, helminth-driven quantitative and qualitative modifications in the overall make-up of gut microbial populations have been proposed to contribute to the immune-suppressive properties of parasites [2-7]. For instance, in a milestone study conducted by Broadhurst et al. [8], experimental infections of a primate model of chronic idiopathic diarrhoea (CID) with the human large intestinal whipworm, Trichuris trichiura, were followed by a significant improvement of clinical signs and weight gain; these were accompanied by a notable increase in microbial alpha diversity in the colonic mucosal microbiota of worm-infected macaques. This observation led the authors to speculate that the onset of Th2-mediated host immune responses against the parasites might have resulted in significant changes of the mucosal environment, such as a reduced bacterial attachment to the intestinal mucosa post-worm colonisation and significant contractions of populations of potentially pathogenic bacteria to levels comparable to those of healthy controls [8]. Another study conducted in a cohort of human volunteers with Coeliac Disease (CeD) experimentally infected with the hookworm of the small intestine, Necator americanus, reported increases in gut microbial richness (observed in both faecal samples and biopsy tissues) that followed parasite colonisation and subsequent challenge with increasing doses of gluten $[4,5,9]$; whilst the detected 
differences did not reach statistical significance (likely due to sample size limitations), the increased gluten tolerance observed in infected CeD volunteers was postulated to contribute to the antiinflammatory properties of $N$. americanus via the restoration of microbial and immune homeostasis [5, 9]. These data point towards a possible role of helminth-associated changes in gut microbial community composition and function in parasite-mediated suppression of chronic inflammation; nonetheless, thus far, studies of the role(s) that the gut microbiota plays in the therapeutic properties of helminth parasites have been carried out in human models of gut diseases [5, 9]. Consequently, the dysbiotic state of the gut microbiota of these individuals at baseline makes the determination of the mechanisms of microbiotadriven helminth immune-suppression challenging.

Nevertheless, recently, the therapeutic properties of controlled infections with $N$. americanus have been investigated in a double-blinded, randomised, placebo-controlled clinical trial conducted in a cohort of 73 human patients with relapsing multiple sclerosis (RMS) (i.e. Worms for Immune Regulation in Multiple Sclerosis, WIRMS; NCT01470521; 10) MS is an autoimmune disease of the central nervous system (CNS) characterised by inflammation, demyelination, and subsequent neural damage [reviewed by 11]. Current, long-term immunosuppressive therapies for MS are often associated with severe side effects and patients will often experience substantial and tragic neurological disabilities related to the disease [reviewed by 11]. The ability of $N$. americanus to stimulate a systemic Th2-dominated environment in the human host represented the main rationale of this clinical trial [12]. Notably, the clinical outcome of the WIRMS study provided further support to the promise of helminth-based therapy for treatment of RMS; indeed, at the end of the study, $51 \%$ of RMS patients experimentally infected with $N$. americanus showed no detectable new CNS lesions, as assessed by magnetic resonance imaging (MRI) scans, vs. $28 \%$ of placebo-treated volunteers [10]. Underpinning the immune-modulatory properties of $\mathrm{N}$. americanus infections, the percentages of eosinophils and of $C D 4+C D 25^{\text {high }} C D 127^{\text {neg }} T$ cells in peripheral blood of worm-colonised individuals was significantly increased 9 months post-infection compared to placebotreated subjects [10]. Given the existence of robust communications between the gut and the CNS by means of immunological, neural and endocrine mechanisms (i.e. gut-systemic-CNS axis; Rhee et al., 2009), as well as recent evidence demonstrating causal relationship between gut microbiome phenotype and the onset of MS [13], it is conceivable that the beneficial properties of $N$. americanus in RMS might be associated, at least in part, to the direct or indirect effects that the parasites exert on the composition of the gut microbiota and relative abundances of individual bacterial species. Thus, building on the availability of unique biological specimens (i.e. faecal samples) collected over the course of the WIRMS trial, we explore, for the first time, the longitudinal changes in gut microbial profiles of human volunteers with RMS, prior to and following experimental infection with $N$. americanus, and subsequent administration of anthelmintic treatment, and compare the findings with data obtained from a cohort of uninfected, placebo-treated RMS patients. In particular, we show that, unlike the gut microbiota of placebo-treated RMS patients, that of $N$. americanus-infected volunteers was characterised by unaltered microbial diversity throughout the course of the trial, and by significant expansions in populations of bacteria with known immune-modulatory properties (e.g. Tenericutes/Mollicutes) with potential roles in parasite-mediated suppression of autoimmunity. 


\section{Materials And Methods \\ Ethics statement}

This phase 2, single centre, randomised, double-blinded, placebo-controlled clinical trial (WIRMS; Clinicaltrials.gov identifier NCT00630383) aimed to assess the therapeutic efficacy of live hookworm (N. americanus) infective larvae in patients with RMS [10]. The trial was conducted at the Queen's Medical Centre, University of Nottingham, UK. The study was approved and carried out in strict accordance and compliance with the National Research Ethics Service Committee East Midlands (reference 11/EM/0140). Written informed consent was obtained from all subjects enrolled in the study.

\section{Trial design}

For details of patient recruitment, inclusion and exclusion criteria and trial design we refer to the original publication by Tanasescu et al. [10]. Briefly, a total of 73 clinically stable RMS patients aged 18-64 (51 females and 22 males), who suffered at least 1 relapse over the prior 12 months or two over the prior 24 months and who were not subjected to immunomodulatory treatment were randomised and assigned to the two treatment groups, i.e. percutaneous infection with $25 \mathrm{~N}$. americanus infective third-stage larvae $(\mathrm{N}+; \mathrm{n}=36)$, or placebo treatment with pharmacopoeial grade water (PBO; $\mathrm{n}=37)$. Stool samples were collected one week prior to infection/placebo-treatment ( $=T_{\text {pre }}$ ), as well as 1 (T1), 5 (T5), and 9 (T9) months post-infection/placebo-treatment (together referred to as $T_{\text {treatment }}$ ), and two months postanthelminthic treatment $\left(=\mathrm{T}_{\text {post }}\right)$ and stored at $-20^{\circ} \mathrm{C}$ until nucleic acids extraction (Fig. 1). Only study subjects who provided samples for all of these time points were included in this study $(n=50)$. Infections were confirmed for each $\mathrm{N}$ + patient via PCR and qPCR-guided $\mathrm{N}$. americanus DNA detection performed using the latest available faecal sample prior to anthelmintic treatment, and following previously established protocols $[10,14,15]$. Two volunteers did not complete the WIRMS trial and thus were excluded from this study.

\section{DNA extraction and bacterial 16S rRNA Illumina sequencing}

Genomic DNA was extracted directly from each faecal content sample, using the PowerSoil@ DNA Isolation Kit (MO BIO Laboratories, Carlsbad, CA, USA), according to manufacturers' instructions, within 1 month from sample collection. High-throughput sequencing of the V3-V4 hypervariable region of the bacterial 16S rRNA gene was performed on an Illumina MiSeq platform according to the standard protocols with minor adjustments. Briefly, the V3-V4 region was PCR-amplified using universal primers [16], that contained the Illumina adapter overhang nucleotide sequences, using the NEBNext hot start high-fidelity DNA polymerase (New England Biolabs), $2 \mathrm{ng} / \alpha$ l of template DNA and the following thermocycling protocol: 2 min at $98^{\circ} \mathrm{C}, 20$ cycles of $15 \mathrm{~s}$ at $98^{\circ} \mathrm{C}-30 \mathrm{~s}$ at $63^{\circ} \mathrm{C}-30 \mathrm{~s}$ at $72{ }^{\circ} \mathrm{C}$, and a final elongation step of $5 \mathrm{~min}$ at $72{ }^{\circ} \mathrm{C}$. Amplicons were purified using AMPure XP beads (Beckman Coulter) and the NEBNext hot start high-fidelity DNA polymerase was used for the index PCR with Nextera $\mathrm{XT}$ index primers (Illumina) according to the following thermocycling protocol: $3 \mathrm{~min}$ at $95^{\circ} \mathrm{C}, 8 \mathrm{cycles}$ of 
$30 \mathrm{~s}$ at $95^{\circ} \mathrm{C}-30 \mathrm{~s}$ at $55^{\circ} \mathrm{C}-30 \mathrm{~s}$ at $72{ }^{\circ} \mathrm{C}$, and $5 \mathrm{~min}$ at $72{ }^{\circ} \mathrm{C}$. The indexed samples were purified using AMPure XP beads, quantified using the Qubit dsDNA high sensitivity kit (Life Technologies), and equal quantities from each sample were pooled. The resulting pooled library was quantified using the NEBNext library quantification kit (New England Biolabs) and sequenced using the v3 chemistry (301 bp paired-end reads). Raw sequence data are available from the Mendeley database at DOI: 10.17632/pkk4vtc57r.1.

\section{Bioinformatics and statistical analyses}

Raw paired-end Illumina reads were trimmed for $16 \mathrm{~S}$ rRNA gene primer sequences using Cutadapt (https://cutadapt.readthedocs.org/en/stable/) and sequence data were processed using the Quantitative Insights Into Microbial Ecology 2 (QIIME2-2019.1; https://qiime2.org) software suite [17]. Successfully joined sequences were quality filtered, dereplicated, chimeras identified, and paired-end reads merged in QIIME2 using DADA2 [18]. Sequences were clustered into Operational Taxonomic Units (OTUs) on the basis of similarity to known bacterial sequences available in the SILVA reference database (https://www.arb-silva.de/download/archive/qiime; Silva_132) sequences that could not be matched to references in the SILVA database were clustered de novo based on pair-wise sequence identity ( $99 \%$ sequence similarity cut-off). The first selected cluster seed was considered as the representative sequence of each OTU. The OTU table with the assigned taxonomy was exported from QIIME2 alongside a weighted UniFrac distance matrix. Singleton OTUs were removed prior to downstream analyses. Cumulative-sum scaling (CSS) was applied, followed by log2 transformation to account for the nonnormal distribution of taxonomic counts data. Statistical analyses were executed using the Calypso software [19] (cgenome.net/calypso/); samples were ordinated in explanatory matrices using supervised Canonical Correspondence Analysis (CCA) including 'infection status' as explanatory variables. Differences in bacterial alpha diversity (Shannon index) between study groups ( $\mathrm{N}+$ and PBO) were evaluated based on rarefied data (read depth of 8 712) and using analysis of variance (ANOVA); F-Tests were used to statistically assess the equality of assessed means (i.e. effect size). To take into account the paired nature of samples from $\mathrm{N}+$ and PBO across timepoints, differences between these sets were assessed using linear mixed effect regression. Differences in beta diversity (weighted UniFrac distances) were identified using Analysis of Similarity (ANOSIM) and effect size indicated by an R-value (between 1 and $+I$, with a value of 0 representing the null hypothesis [20]). Differences in the abundance of individual microbial taxa between groups were assessed using the Linear Discriminant Analysis Effect Size (LEfSe) workflow (21). Bacterial taxa predictive of the study cohort either responding to hookworm treatment $\left(\mathrm{N}+{ }_{\text {responders }}\right)$ or having MS disease activity $\left(\mathrm{N}+{ }_{\text {non-responders }}\right)$ were identified via Wilcoxon rank test (19). The discriminatory power of microbial community profiles to distinguish between two biological conditions is characterized by a Support Vector Machine evaluated by leave-one-out crossvalidation [19]. The discriminatory power of individual taxa can be assessed via Area Under the ROC Curve (AUC; 1 = good measure of separability, $0=$ poor measure of separability), odds ratio $([+/-] 1=$ odds of one event being the same in either the presence or absence of the other event, $>[+/-] 1=$ the presence of one event increases/reduces the odds of the other event) [19].

\section{Results}




\section{The faecal microbiota composition of parasite-infected RMS patients differs from that of placebo-treated RMS volunteers}

A total of 226 faecal samples from 50 WIRMS study subjects (i.e. 36 females and 14 males) who met the inclusion criteria (see Material and Methods and [10]) were analysed for microbiota profiling as described below. Following data and metadata unblinding at the end of the trial [cf. 10], it was established that 24 of these subjects had been percutaneously infected with $25 \mathrm{~N}$. americanus infective larvae $(\mathrm{N}+, 17$ females and 7 males), whilst 26 had been placebo-treated with PBO (19 females and 7 males). Individual faecal samples collected from these subjects 1 week prior to experimental infection/placebo treatment (p.i./p.; T pre), and at 1, 5 and 9 months p.i./p. (T1, T5 and T9, respectively; samples together referred to as ' $T_{\text {treatment }}$ ') and 2 months post-anthelmintic treatment ( $T_{\text {post }} ;$ Fig. 1) were subjected to high-throughput (Illumina) sequencing of the bacterial 16S rRNA gene fragment. This yielded a total of 16158693 (per sample mean: $68180 \pm 70$ 000) paired-end reads; of these, 9100255 high-quality sequences (per sample mean $38397 \pm 31519$ ) were retained following quality control. Rarefaction curves generated following in silico subtraction of low-quality sequences indicated that the majority of faecal bacterial communities were represented in the remaining sequence data, thus allowing us to undertake further analyses (Fig. S1).

These high-quality sequences were assigned to a total of 5611 OTUs and 14 bacterial phyla (data available from Mendeley Data at DOI: 10.17632/pkk4vtc57r.1). The phyla Bacteroidetes ( $+=43.8 \%$ average $\pm 0.4 \%$ standard deviation, and PBO $=52.2 \% \pm 1.6 \%$, respectively $)$ and Firmicutes $(\mathrm{N}+=51 \% \pm$ $0.3 \%$, and $\mathrm{PBO}=43.7 \% \pm 0.3 \%$, respectively) were predominant in all samples analysed, followed by the phyla Proteobacteria $(\mathrm{N}+=2.4 \% \pm 1.4 \%$, and $\mathrm{PBO}=2.2 \% \pm 2 \%$, respectively), Actinobacteria $(\mathrm{N}+=2.3 \% \pm$ $1.3 \%$, and $\mathrm{PBO}=1.8 \% \pm 1.2 \%$, respectively), and Tenericutes $(\mathrm{N}+=0.5 \% \pm 3 \%$, and $\mathrm{PBO}=0.1 \% \pm 3.1 \%$, respectively; Fig. 2A). Faecal microbial community profiles were ordinated by CCA that significantly separated samples by infection status over the course of infection ( $P=0.001$; Fig. $2 B$ ). No statistically significant differences in bacterial community composition were detected pre-infection and postanthelmintic treatment between the two study cohorts $(P=0.885, P=0.283$ respectively; Fig. $2 B)$, or between female and male study subjects at $T_{\text {pre, }} T_{\text {treatment, }}$ and $T_{\text {post }}$ (Fig. S2).

\section{Infection with Necator americanus is associated with stable gut microbial alpha diversity}

No significant differences in microbial alpha diversity, measured through the Shannon index, were detected between the faecal microbiota of $\mathrm{N}+$ and $\mathrm{PBO}$ patients pre-hookworm infection (effect size $(\mathrm{F})=$ 0.19, $\mathrm{P}=0.67$; Fig. 3A). Nevertheless, following experimental infection, Shannon diversity was significantly elevated in $\mathrm{N}+$ compared to $\mathrm{PBO}$ subjects $(\mathrm{F}=5.2, \mathrm{P}=0.025$; Fig. $3 \mathrm{~A})$; this predominantly resulted from increased microbiota evenness $(F=6.5, P=0.012)$, rather than richness $(F=2.6, P=0.11$; 
Fig. S3). Additionally, Shannon diversity was significantly decreased ( $F=5.7, P=0.027$; Fig. S4 and Fig. $\mathrm{S} 5)$ in PBO patients who had suffered clinical relapses over the course of the trial $\left(\mathrm{PBO}_{\text {relapse }} ; \mathrm{n}=6\right)$ compared to ones that did not $\left(\mathrm{PBO}_{\text {no-relapse }} \mathrm{n}=21\right)$. The trend in $\mathrm{N}+$ patients who had suffered relapses $\left(\mathrm{N}+{ }_{\text {relapse; }} \mathrm{n}=3\right)$ compared to patients that did not was less obvious $\left(\mathrm{N}+{ }_{\text {no-relapse; }} \mathrm{n}=21 ; \mathrm{Fig}\right.$. $\mathrm{S} 4$ and Fig. S5). Notably, significant differences in microbial diversity between the two study cohorts were no longer detected following anthelmintic treatment $(F=2.3, P=0.14$; Fig. $3 A)$. In addition, no significant change in faecal microbial Shannon diversity was detected in $N+$ patients over the course of the trial, whilst this was decreased, albeit marginally, in faecal samples from $P B O$ subjects post placebo treatment $(F=5.3, P$ $=0.04 ;$ Fig. 3B). No significant differences in gut microbial beta diversity between $\mathrm{N}+$ and PBO patients (Fig. S6A), and over time for either cohort (Fig. S6B), were observed at any point throughout this study.

\section{Tenericutes/Mollicutes are expanded in the faecal microbiota of parasite-infected RMS volunteers}

LEfSe analysis revealed significant differences in the relative abundances of individual microbial taxa (phylum to species level) between $\mathrm{N}+$ and PBO patients at $\mathrm{T}_{\text {pre, }} \mathrm{T}_{\text {treatment, }}$ and $\mathrm{T}_{\text {post }}$ (Fig. 4), as well as between the two groups at each timepoint within $\mathrm{T}_{\text {treatment }}$ (Fig. S7A). Of these bacterial taxa, five genera (Roseburia, Dorea, Tyzzerella, Fusicatenibacter, and Agathobacter) belonging to the family Lachnospiraceae, Peptostreptococcaceae, Carnobacteriaceae, and Coriobacteriaceae were significantly more abundant in PBO subjects compared to $\mathrm{N}+$ subjects over the course of helminth infection $\left(T_{\text {treatment }}\right)$. Conversely, three orders (RF39, Izimaplasmatales, and Anaeroplasmatales) belonging to Tenericutes/Molicutes, the families VadinBB60, Clostridiaceae (genus Hungatella), Ruminococcaceae (genera UCG005 and UCG010), Flavobacteriaceae, and the genera Sutterella, Barnsiella, and Coprobacter were significantly more abundant in the faecal microbiota of $\mathrm{N}+$ subjects than in that of PBO (Fig. 4). Notably, differences in Mollicutes abundance resulted from expanded populations of these bacteria in the microbiota from $\mathrm{N}+$ and a simultaneous contraction of the same taxa in faecal samples from PBO (Fig. S7B). Finally, Coriobacteriaceae remained more abundant in the microbiota of PBO subjects at $\mathrm{T}_{\text {post }}$ whilst Tenericutes/Mollicutes and VadinBB60, remained more abundant in $\mathrm{N}+$ subjects at the same time point (Fig. 4).

A summary of the findings from this study, including populations of gut bacteria expanded or reduced upon experimental infection with N. americanus, as well as fluctuations in gut microbial alpha- and betadiversity over the course of the WIRMS trial, is available from the MICrobiome HELminth INteraction database (MICHELINdb) at www.michelindb.com [22].

\section{Relapses are associated with differences in the abundances of selected bacterial populations in parasite-infected RMS volunteers}


Differences in gut microbiota composition between $\mathrm{N}+$ patients who presented significant MRI activity throughout the study $\left(\mathrm{N}+{ }_{\text {non-responders }} \mathrm{n}=10\right)$ vs. $\mathrm{N}+$ who did not show disease activity $(\mathrm{N}+$ responders; $=14$ ) [cf. 10] were also investigated. Whilst no substantial differences in overall microbiota composition were detected between faecal samples from these sub-cohorts at $T_{\text {pre }}$ and $T_{\text {post }}$ (Figs. S8 and 5A), CCA analysis at $T_{\text {treatment }}$ detected significant differences between the microbial profiles of $\mathrm{N}+{ }_{\text {non-responders }}$ and $\mathrm{N}+$ responders $(P=0.001$; Fig. $5 B)$. No significant differences in Shannon and beta diversity were detected between these groups at any timepoint (Figs. S9 and S10); nevertheless, gut microbial beta diversity was significantly elevated in the $\mathrm{N}+{ }_{\text {responders }}$ cohort at $\mathrm{T}_{\text {treatment, }}$ albeit with a small effect size $(R=0.08, P=0.001$; Fig. 6). This difference resulted from a significant increase in beta diversity in the faecal microbiota of $\mathrm{N}+{ }_{\text {responders, }}$ as well as a decrease in that of $\mathrm{N}+{ }_{\text {non-responders }}$ (Fig. S11). Differences in the relative abundances of individual microbial taxa (phylum to species level) between the faecal microbiota of $\mathrm{N}+{ }_{\text {non-responders }}$ and $\mathrm{N}+{ }_{\text {responders }}$ patients were determined via LEfSe (Fig. 7). Amongst others, the bacterial families Defluviitaleaceae (UCG011), Ruminococcaceae (Ruminiclostridium and UCG004), Christensenellaceae (R7), Eubacteriaceae (E. coprostanoligenes), Flavobacteriaceae, Porphyromonadaceae (Parabacteroides), and Anaeroplasmataceae were significantly more abundant in the faecal microbiota of $\mathrm{N}+$ responders, compared to that of $\mathrm{N}+{ }_{\text {non-responders }}$ at $\mathrm{T}_{\text {treatment }}$ (Fig. 7 ), whilst the opposite trend was observed for Eubacteriaceae (E. xylanophilum and E. ruminantium), Ruminococcaceae (Anaerofilum, UCG003, Ruminococcus), Lachnospiraceae (Roseburia, Tyzerella, UCG010, NK4A136), and Prevotellaceae (Paraprevotella and Prevotella) (Fig. 7). Analysis of bacterial taxa associated with a positive outcome of hookworm treatment in RMS, yielded levels of Parabacteroides as

the best predictor for $\mathrm{N}+{ }_{\text {responders }}$ (high levels) and $\mathrm{N}+{ }_{\text {non-responders }}$ (low levels) cohorts. Parabacteroides also remained the best predictor at $T_{\text {treatment, }}$ with the abundances of Roseburia NK4A136, Ruminococcus, Oscillibacter Eubacterium (coprostanoligenes) also proving to be associated to treatment outcome in RMS patients (Fig. 8).

\section{Discussion}

In the present MHRA approved study we investigated, for the first time, the quantitative and qualitative changes in gut microbial profiles of human volunteers with RMS prior to and following experimental infection with $N$. americanus, and following administration of anthelmintic treatment, and compared the findings with data obtained from an age- and gender-matched cohort of RMS patients subjected to placebo treatment.

\section{Changes in gut microbial diversity}

No substantial differences in gut microbiota composition were detected between $\mathrm{N}+$ and $P B O$ patients prior to experimental infection based on CCA analysis, while significant alterations in faecal microbial profiles were detected in the infected cohort compared with uninfected subjects post-worm colonisation. Of note, these differences were no longer significant post-anthelminthic treatment, thus suggesting a 
direct modulating effect of live hookworm infection on the microbial communities inhabiting the host gut. In particular, microbial alpha diversity was significantly higher in the gut of $\mathrm{N}+$ subjects compared to $P B O$ over the course of helminth treatment. It must however be pointed out that this observation was predominantly linked to a marked decrease of gut microbial alpha diversity, and specifically of bacterial evenness, in $P B O$ over time. Parasite removal via anthelmintic treatment did not result in microbial alpha diversity alterations in both $N+$ and $P B O$. Notably, compared with values recorded prior to hookworm infection, this parameter was significantly increased in $N+$ subjects at end the of the trial, whilst the opposite trend was observed in the gut microbiota of $P B O$. This finding is of particular interest, since elevated levels of microbial alpha diversity are typically associated with a 'healthier' gut microbiome and overall host health [reviewed by 23]. Similarly, increases in gut microbial alpha diversity were reported in CeD subjects experimentally infected with $N$. americanus, which led the authors to speculate that this mechanism might be at least partially responsible for the therapeutic effect of deliberate helminth infections in individuals affected by selected allergic and autoimmune disorders [5, 9]. On the other hand, a decrease in gut microbial alpha diversity has previously been reported during MS relapses [24]. This matches our observations of a significantly lower microbial alpha diversity being detected at $T_{\text {treatment }}$ in the faecal microbiota of $P B O$ volunteers who suffered relapses over the course of the trial, as well as decreased alpha diversity following specific relapse events in both $N t_{\text {relapse }}$ and $P B O_{\text {relapse }}$ patients.

\section{Gut microbial taxa expanded in the $P B O$ cohort}

The relative abundances of several gut bacterial taxa were also significantly altered in $N+$ subjects compared with the $P B O$ cohort, both prior to $N$. americanus experimental infections and, more markedly, post-helminth colonisation. Indeed, despite subject randomisation prior to the beginning of the study, we detected minor differences in gut microbial composition between study cohorts at $\mathrm{T}_{\text {pre. }}$. No prior characterisation of faecal microbial communities was conducted prior to group assignments; in addition, due to the intrinsic heterogeneity of gut microbial communities across any given population $[25,26]$, differences in the abundances of gut microbial taxa are frequently detected between groups of individuals enrolled in randomized, double-blinded, placebo-controlled trials [27, 28], with varying functional significance [28]. Nevertheless, in our study, most differences between $\mathrm{N}+$ and $P B O$ gut microbial profiles were detected post-infection, peaking at 9 months post-helminth colonisation. Notably, in $P B O$ patients, bacterial taxa that have been previously associated with the gut microbiota of relapsing MS patients [24] were significantly expanded; in particular, a family of anaerobic bacteria, the Lachnospiraceae, including the genera Roseburia, Dorea, and Tyzzerella (amongst others), were significantly increased in the faecal microbiota of $P B O$ compared to $N+$ subjects post-infection. Lachnospiraceae is a key family of the human gut microbiome that degrades complex polysaccharides to short-chain fatty acids (SCFAs), i.e. acetate, butyrate, and propionate, that are used for energy by the host. Notably, SCFAs are known for their anti-inflammatory properties [29]. This is of note, since Lachnospiraceae have been reported to be substantially expanded in the gut microbiota of individuals affected by pathological conditions, such as inflammatory bowel disease (IBD) [30]. In particular, the 
abundance of Dorea increases in the gut of irritable bowel syndrome patients [31], as well as during intestinal inflammation [32]. Whilst Dorea are known to produce the SCFA butyrate, this genus of bacteria also metabolises sialic acids, which are commonly found at the terminal ends of mucins; release of these acids is implicated in mucin degradation, with consequences ranging from increased gut permeability to compromised gut homeostasis; this cascade of events has been suggested to result in proinflammatory responses that promote chronic inflammation in MS [33,34]. The expansion of Lachnospiraceae and its respective genera in the $P B O$ cohort over the course of the trial is likely associated to MS disease progression and an immune shift towards a pro-inflammatory phenotype [34]. Of note, no significant expansion of Lachnospiraceae could be detected in the gut microbiota of $N+$ (as a whole) over time, with the exception of $N+{ }_{n}$ non-responders (see below).

\section{Gut microbial taxa expanded in the $\mathbf{N +}$ cohort}

Bacteria belonging to the phylum Tenericutes were substantially and consistently increased in the gut microbiota of $\mathrm{N}+$ patients post-infection. This phylum consists of the sole class Mollicutes, Gramnegative, small and wall-less bacteria that fulfil a diverse array of roles within the mammalian microbiome [reviewed by 35,36$]$. The relative abundance of these bacteria has been reported to differ between the normal gut microbiota and that found in a wide range of autoimmune conditions, such as IBD [37, 38], Type 1 Diabetes [39-41], MS [42], and experimental autoimmune encephalomyelitis (EAE; a murine model of MS) [43]. Albeit inconsistently [40,41], Tenericutes/Mollicutes are often reduced in the gut microbiota of the diseased cohort, when compared to healthy controls [37-39, 42, 43]. Notably, whilst one of these studies reported higher Tenericutes abundance in the gut microbiota of healthy controls than in paediatric MS patients, exposure to immunomodulatory drugs reversed this trend [42]. Indeed, these bacteria have been suggested to proliferate in Th2-dominant environments [8, 44]. Tenericutes/Mollicutes were also expended in the gut microbiota of vertebrates infected by Th2-inducing helminth parasites, human cohorts naturally infected with roundworms (i.e. Trichuris and/or Ascaris and/or hookworm) [45], rats infected with the tapeworm Hymenolepis diminuta [44], and primates with CID experimentally infected with T. trichiura [8]. This is in accordance with data from the WIRMS trial, that reported a markedly increased eosinophilia in $N+$ compared to $P B O$ subjects [10]. Nevertheless, the functional consequences that expanded populations of Tenericutes/Mollicutes associated to infection by parasitic helminths play in the pathophysiology of the abovementioned chronic inflammatory and autoimmune disorders, as well as in RMS, remains to be determined. Alongside the evidence from previous studies, our data lends further support to the need for mechanistic investigations of the interactions between helminth and/or their products and this bacterial taxon in vitro and/or in vivo, to assist untangling its potential role in disease progression and prevention.

\section{Gut microbial differences between $\mathbf{N} t_{\text {responders }}$ and $\mathbf{N} \boldsymbol{t}_{\text {non- }}$} responders 
In addition, we investigated the differences in gut microbial composition between $\mathrm{N}+_{\text {responders }}$ and $\mathrm{N}+_{\text {non- }}$ responders, with the aim to identify potential bacterial candidates with roles in MS disease activity. Whilst the overall gut microbiota of these sub-cohorts did not differ substantially prior to helminth infection and or post-anthelmintic treatment, differences were recorded in the gut microbiota of these sub-groups posthelminth colonisation. In particular, while gut microbial alpha diversity remained similar between $\mathrm{N}_{\text {responders }}$ and $\mathrm{N}_{\text {non-responders, }}$, beta diversity was significantly higher in the former (albeit with a small effect size), indicating a 'diversified' gut microbiota in $N t_{\text {responders, }}$ either directly associated to $N$. americanus infection and/or, indirectly, to the immune responses mounted against the worms [1]. Meanwhile, the gut microbial beta diversity of $\mathrm{N}$ non-responders was reduced over the course of the trial, possibly resulting from downstream effects of disease progression on the host gut microbiota [10, 46-48].

We detected significantly increased populations of Tenericutes/Mollicutes in the gut microbiota of $\mathrm{N}_{\text {responders, }}$ and expanded populations of Lachnospiraceae in $\mathrm{N}_{\text {non-responders, }}$ further supporting the hypothesis of a functional role of these taxa in the immune-modulatory properties of $\mathrm{N}$. americanus. Furthermore, Flavobacteriaceae, the largest family in the phylum Bacteroidetes [49], was consistently increased in the gut microbiota of $\mathrm{Nt}_{\text {responders. }}$. This taxon has been repeatedly reported to be depleted in people suffering from autoimmune conditions, such as rheumatoid arthritis [50] and myasthenia gravis [51], although the functional importance of this taxon in the pathophysiology of these conditions remains elusive. Finally, in this study, we asked the question of whether some of the minor differences in gut microbial profiles observed between $\mathrm{N} t_{\text {responders }}$ and $\mathrm{N}$ non-responders $_{\text {nior }}$ pro hookworm experimental infection might be associated with positive or negative clinical outcomes. Amongst others, the genus Parabacteroides was significantly more abundant in the gut microbiota of $\mathrm{Nt}_{\text {responders }}$ compared to

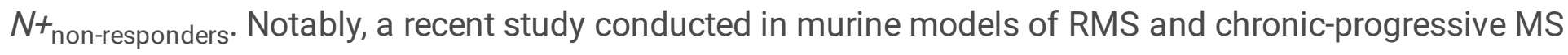
identified Parabacteroides as more abundant in the gut microbiota of control mice [52]. Additionally, a previous study detected a significant reduction of Parabacteroides populations in the faecal microbiota of $71 \mathrm{MS}$ patients not undergoing immunesuppressive treatment compared to that of 71 healthy control subjects [53]. Subsequent monocolonisation of antibiotic-treated mice with Parabacteroides distasonis led to significant increases in the CD4+IL-10+ T lymphocyte population in mesenteric lymph nodes and spleens [53]. Furthermore, stimulation of peripheral blood mononuclear cells (PBMCs) from MS patients or healthy controls with total bacterial extracts isolated from the stool samples of the same subjects resulted in the inability of PBMCs from MS patients to differentiate or expand CD25+FoxP3+ Treg populations [53]. This observation led the authors to hypothesise that prior exposure to $P$. distasonis or other "beneficial" bacteria may have contributed to the expanding regulatory $\mathrm{T}$ lymphocyte precursor populations in mice, hence promoting anti-inflammatory responses upon subsequent exposure to the same bacteria [53]. Furthermore, it is known that Parabacteroides metabolise phytoestrogens [5456]. Estrogens have been shown to possess disease-suppressive properties in MS as evidenced in several studies in humans and animal models [57].

\section{Conclusion}


In conclusion, we detected significant qualitative and quantitative differences in gut microbial composition between RMS subjects experimentally infected with N. americanus and placebo-treated individuals. A significant decrease in microbial alpha diversity, accompanied by expanded populations of Lachnospiraceae were detected in the faecal microbiota of the PBO cohort, in accordance with published data investigating gut microbial composition during MS relapses. Significantly higher microbial alpha diversity in the gut microbiota of $\mathrm{N}+$ patients suggest improved gut microbial and homeostasis. This was further supported by increased relative abundances of Tenericutes. Furthermore, we identified significant differences between the gut microbial profiles of $\mathrm{N}+{ }_{\text {responders }}$ and $\mathrm{N}+{ }_{\text {non-responders, }}$ with populations of Parabacteroides being significantly more abundant in the former. Overall, these data lend support to the hypothesis of a contributory role of parasite-associated modulation of host gut microbiota composition to the immune-suppressive properties of hookworms. This work adds valuable knowledge to current understanding of parasite-microbiota associations and will assist future mechanistic studies aimed to unravel the causality of these relationships.

\section{Abbreviations}

ANOSIM

Analysis of similarity

CCA

Canonical correlation analysis

CSS

Cumulative-sum scaling

GI

Gastrointestinal

LEfSe

Linear discriminant analysis effect size

MS

Multiple sclerosis

$\mathrm{N}+$

Hookworm infected

OTU

Operational taxonomic unit

PCoA

Principal coordinates analysis

PBO

Placebo

RDA

Redundancy analysis

\section{Declarations}




\section{Ethics approval and consent to participate}

Ethical approval was obtained from East Midlands National Research Ethics Committee (reference 11/EM/0140) and informed consent secured from all patients.

\section{Consent for publication}

Not applicable

\section{Availability of data and material}

The 16S rRNA gene sequence datasets generated and analyzed during the current study are available from Mendeley Data (DOI: 10.17632/pkk4vtc57r.1).

\section{Competing interests}

The authors declare that they have no competing interests.

\section{Funding}

The clinical trial from which specimens analysed in this study were derived was supported by the MS Society of the Great Britain and Northern Ireland, the Forman Hardy Charitable Trust via the University of Nottingham and an unrestricted grant from Bayer-Schering awarded to DIP and CSC. T.P.J. is the grateful recipient of a PhD scholarship by the Biotechnology and Biological Sciences Research Council (BBSRC) of the United Kingdom. Research in the C.C. laboratory is funded by grants by the Isaac Newton Trust, the Isaac Newton Trust/Wellcome Trust/ University of Cambridge joint grant scheme and by the Royal Society (UK).

\section{Author's contributions}

CC, CCo, and DP conceived the project. TPJ, MP, RT, and GT collected the samples and TPJ processed the samples for amplicon sequencing. TPJ performed bioinformatic analysis on the amplicon data. TPJ and CC interpreted the data and drafted the work for publication. All authors read and approved the final manuscript. 


\section{Acknowledgements}

Not applicable.

\section{References}

1. Brosschot TP, Reynolds LA. The impact of a helminth-modified microbiome on host immunity. Mucosal Immunol. 2018.

2. Bach JF. The hygiene hypothesis in autoimmunity: the role of pathogens and commensals. Nat Rev Immunol. 2017;18(2):105-20.

3. Giacomin P, Croese J, Krause L, Loukas A, Cantacessi C. Suppression of inflammation by helminths: a role for the gut microbiota? Philos Trans R Soc Lond B Biol Sci. 2015;370(1675).

4. Cantacessi C, Giacomin P, Croese J, Zakrzewski M, Sotillo J, McCann L, et al. Impact of experimental hookworm infection on the human gut microbiota. J Infect Dis. 2014;210(9):1431-4.

5. Giacomin P, Zakrzewski M, Croese J, Su X, Sotillo J, McCann L, et al. Experimental hookworm infection and escalating gluten challenges are associated with increased microbial richness in celiac subjects. Sci Rep. 2015;5:13797.

6. Mutapi F. The gut microbiome in the helminth infected host. Trends Parasitol. 2015;31(9):405-6.

7. Ramanan D, Bowcutt R, Lee SC, Tang MS, Kurtz ZD, Ding Y, et al. Helminth infection promotes colonization resistance via type 2 immunity. Science. 2016;352(6285):608-12.

8. Broadhurst MJ, Ardeshir A, Kanwar B, Mirpuri J, Gundra UM, Leung JM, et al. Therapeutic helminth infection of macaques with idiopathic chronic diarrhea alters the inflammatory signature and mucosal microbiota of the colon. PLoS Pathog. 2012;8(11):e1003000.

9. Giacomin P, Zakrzewski M, Jenkins TP, Su X, Al-Hallaf R, Croese J, et al. Changes in duodenal tissueassociated microbiota following hookworm infection and consecutive gluten challenges in humans with coeliac disease. Sci Rep. 2016;6:36797.

10. Tanasescu R, Tench CR, Constantinescu CS, Telford G, Singh S, Frakich N, et al. Worms for immune regulation of multiple sclerosis (WIRMS): a randomised double-blinded placebo controlled trial. JAMA Neurology. 2020;accepted.

11. Mahad DH, Trapp BD, Lassmann H. Progressive multiple sclerosis 1 Pathological mechanisms in progressive multiple sclerosis. Lancet Neurology. 2015;14(2):183-93.

12. Blount D, Hooi D, Feary J, Venn A, Telford G, Brown A, et al. Immunologic profiles of persons recruited for a randomized, placebo-controlled clinical trial of hookworm infection. Am J Trop Med Hyg. 2009;81(5):911-6.

13. Jangi S, Gandhi R, Cox LM, Li N, von Glehn F, Yan R, et al. Alterations of the human gut microbiome in multiple sclerosis. Nat Commun. 2016;7:12015. 
14. Gasser RB, Chilton NB, Hoste H, Beveridge I. Rapid sequencing of rDNA from single worms and eggs of parasitic helminths. Nucleic acids research. 1993;21(10):2525-6.

15. Pilotte N, Papaiakovou M, Grant JR, Bierwert LA, Llewellyn S, McCarthy JS, et al. Improved PCRBased Detection of Soil Transmitted Helminth Infections Using a Next-Generation Sequencing Approach to Assay Design. PLoS Negl Trop Dis. 2016;10(3):e0004578.

16. Klindworth A, Pruesse E, Schweer T, Peplies J, Quast C, Horn M, et al. Evaluation of general 16S ribosomal RNA gene PCR primers for classical and next-generation sequencing-based diversity studies. Nucleic Acids Res. 2013;41(1):e1.

17. Caporaso JG, Kuczynski J, Stombaugh J, Bittinger K, Bushman FD, Costello EK. QIIME allows analysis of high-throughput community sequencing data. Nature Meth. 2010;7.

18. Callahan BJ, McMurdie PJ, Rosen MJ, Han AW, Johnson AJ, Holmes SP. DADA2: High-resolution sample inference from Illumina amplicon data. Nat Methods. 2016;13(7):581-3.

19. Zakrzewski M, Proietti C, Ellis JJ, Hasan S, Brion MJ, Berger B, et al. Calypso: a user-friendly webserver for mining and visualizing microbiome-environment interactions. Bioinformatics. 2017;33(5):782-3.

20. Clarke KR. Non-parametric multivariate analyses of changes in community structure. Austral Ecology. 1993;18(1):117-43.

21. Segata N, Izard J, Waldron L, Gevers D, Miropolsky L, Garrett WS. Metagenomic biomarker discovery and explanation. Genome Biol. 2011;12.

22. Scotti R, Southern S, Boinett C, Jenkins TP, Cortés A, Cantacessi C. MICHELINdb: a web-based tool for mining of helminth-microbiota interaction datasets, and a meta-analysis of current research. Microbiome. 2020;8(1):10.

23. Lloyd-Price J, Abu-Ali G, Huttenhower C. The healthy human microbiome. Genome Med. 2016;8(1):51.

24. Chen J, Chia N, Kalari KR, Yao JZ, Novotna M, Soldan MM, et al. Multiple sclerosis patients have a distinct gut microbiota compared to healthy controls. Sci Rep. 2016;6:28484.

25. Lozupone CA, Stombaugh JI, Gordon JI, Jansson JK, Knight R. Diversity, stability and resilience of the human gut microbiota. Nature. 2012;489(7415):220-30.

26. Rooks MG, Garrett WS. Gut microbiota, metabolites and host immunity. Nat Rev Immunol. 2016;16(6):341-52.

27. Reijnders D, Goossens Gijs H, Hermes Gerben DA, Neis Evelien PJG, van der Beek Christina M, Most J, et al. Effects of Gut Microbiota Manipulation by Antibiotics on Host Metabolism in Obese Humans: A Randomized Double-Blind Placebo-Controlled Trial. Cell Metabolism. 2016;24(1):63-74.

28. Hjorth MF, Roager HM, Larsen TM, Poulsen SK, Licht TR, Bahl MI, et al. Pre-treatment microbial Prevotella-to-Bacteroides ratio, determines body fat loss success during a 6-month randomized controlled diet intervention. Int J Obes (Lond). 2018;42(3):580-3. 
29. Biddle A, Stewart L, Blanchard J, Leschine S. Untangling the genetic basis of fibrolytic specialization by Lachnospiraceae and Ruminococcaceae in diverse gut communities. Diversity. 2013;5(3):627-40.

30. Png CW, Lindén SK, Gilshenan KS, Zoetendal EG, McSweeney CS, Sly LI, et al. Mucolytic bacteria with increased prevalence in IBD mucosa augment in vitro utilization of mucin by other bacteria. Am J Gastroenterol. 2010;105(11):2420.

31. Rajilić-Stojanović M, Biagi E, Heilig HGHJ, Kajander K, Kekkonen RA, Tims S, et al. Global and Deep Molecular Analysis of Microbiota Signatures in Fecal Samples From Patients With Irritable Bowel Syndrome. Gastroenterology.141(5):1792-801.

32. Jenq RR, Ubeda C, Taur Y, Menezes CC, Khanin R, Dudakov JA, et al. Regulation of intestinal inflammation by microbiota following allogeneic bone marrow transplantation. J Exp Med. 2012;209(5):903-11.

33. Crost EH, Tailford LE, Le Gall G, Fons M, Henrissat B, Juge N. Utilisation of mucin glycans by the human gut symbiont Ruminococcus gnavus is strain-dependent. PLoS One. 2013;8(10):e76341.

34. Shahi SK, Freedman SN, Mangalam AK. Gut microbiome in multiple sclerosis: The players involved and the roles they play. Gut microbes. 2017;8(6):607-15.

35. Brown DR. Tenericutes. Bergey's Manual of Systematics of Archaea and Bacteria. 2015:1-2.

36. Gupta RS, Sawnani S, Adeolu M, Alnajar S, Oren A. Phylogenetic framework for the phylum Tenericutes based on genome sequence data: proposal for the creation of a new order Mycoplasmoidales ord. nov., containing two new families Mycoplasmoidaceae fam. nov. and Metamycoplasmataceae fam. nov. harbouring Eperythrozoon, Ureaplasma and five novel genera. Antonie van Leeuwenhoek. 2018;111(9):1583-630.

37. Imhann F, Vich Vila A, Bonder MJ, Fu J, Gevers D, Visschedijk MC, et al. Interplay of host genetics and gut microbiota underlying the onset and clinical presentation of inflammatory bowel disease. Gut. 2018;67(1):108.

38. Willing BP, Dicksved J, Halfvarson J, Andersson AF, Lucio M, Zheng Z, et al. A Pyrosequencing Study in Twins Shows That Gastrointestinal Microbial Profiles Vary With Inflammatory Bowel Disease Phenotypes. Gastroenterology. 2010;139(6):1844-54.e1.

39. Brown CT, Davis-Richardson AG, Giongo A, Gano KA, Crabb DB, Mukherjee N, et al. Gut Microbiome Metagenomics Analysis Suggests a Functional Model for the Development of Autoimmunity for Type 1 Diabetes. PLOS ONE. 2011;6(10):e25792.

40. Patterson E, Marques TM, O'Sullivan O, Fitzgerald P, Fitzgerald GF, Cotter PD, et al. Streptozotocininduced type-1-diabetes disease onset in Sprague-Dawley rats is associated with an altered intestinal microbiota composition and decreased diversity. Microbiology. 2015;161(1):182-93.

41. Peng J, Narasimhan S, Marchesi JR, Benson A, Wong FS, Wen L. Long term effect of gut microbiota transfer on diabetes development. J Autoimmun. 2014;53:85-94.

42. Tremlett $H$, Fadrosh DW, Faruqi AA, Zhu F, Hart J, Roalstad S, et al. Gut microbiota in early pediatric multiple sclerosis: a case-control study. Eur J Neurol. 2016;23(8):1308-21. 
43. Ooi JH, Waddell A, Lin Y-D, Albert I, Rust LT, Holden V, et al. Dominant Effects of the Diet on the Microbiome and the Local and Systemic Immune Response in Mice. PLoS One. 2014;9(1):e86366.

44. Wegener Parfrey L, Jirku M, Sima R, Jalovecka M, Sak B, Grigore K, et al. A benign helminth alters the host immune system and the gut microbiota in a rat model system. PLoS One. 2017;12(8):e0182205.

45. Lee SC, Tang MS, Lim YA, Choy SH, Kurtz ZD, Cox LM, et al. Helminth colonization is associated with increased diversity of the gut microbiota. PLoS Negl Trop Dis. 2014;8(5):e2880.

46. Clemente JC, Manasson J, Scher JU. The role of the gut microbiome in systemic inflammatory disease. BMJ. 2018;360:j5145.

47. Halfvarson J, Brislawn CJ, Lamendella R, Vázquez-Baeza Y, Walters WA, Bramer LM, et al. Dynamics of the human gut microbiome in inflammatory bowel disease. Nat Microbiol. 2017;2(5):17004.

48. Morgan XC, Tickle TL, Sokol H, Gevers D, Devaney KL, Ward DV, et al. Dysfunction of the intestinal microbiome in inflammatory bowel disease and treatment. Genome Biology. 2012;13(9):R79.

49. McBride MJ. The Family Flavobacteriaceae. In: Rosenberg E, DeLong EF, Lory S, Stackebrandt E, Thompson F, editors. The Prokaryotes: Other Major Lineages of Bacteria and The Archaea. Berlin, Heidelberg: Springer Berlin Heidelberg; 2014. p. 643-76.

50. Picchianti-Diamanti A, Panebianco C, Salemi S, Sorgi ML, Di Rosa R, Tropea A, et al. Analysis of Gut Microbiota in Rheumatoid Arthritis Patients: Disease-Related Dysbiosis and Modifications Induced by Etanercept. Int J Mol Sci. 2018;19(10):2938.

51. Moris G, Arboleya S, Mancabelli L, Milani C, Ventura M, de Los Reyes-Gavilan CG, et al. Fecal microbiota profile in a group of myasthenia gravis patients. Sci Rep. 2018;8(1):14384.

52. Gandy KAO, Zhang J, Nagarkatti P, Nagarkatti M. The role of gut microbiota in shaping the relapseremitting and chronic-progressive forms of multiple sclerosis in mouse models. Sci Rep. 2019;9(1):6923.

53. Cekanaviciute E, Yoo BB, Runia TF, Debelius JW, Singh S, Nelson CA, et al. Gut bacteria from multiple sclerosis patients modulate human T cells and exacerbate symptoms in mouse models. Proc Natl Acad Sci U S A. 2017;114(40):10713-8.

54. Schogor AL, Huws SA, Santos GT, Scollan ND, Hauck BD, Winters AL, et al. Ruminal Prevotella spp. may play an important role in the conversion of plant lignans into human health beneficial antioxidants. PloS One. 2014;9(4):e87949.

55. Maruo T, Sakamoto M, Ito C, Toda T, Benno Y. Adlercreutzia equolifaciens gen. nov., sp. nov., an equol-producing bacterium isolated from human faeces, and emended description of the genus Eggerthella. International Journal of Systematic and Evolutionary Microbiology. 2008;58(5):1221-7.

56. Tsuchihashi R, Sakamoto S, Kodera M, Nohara T, Kinjo J. Microbial metabolism of soy isoflavones by human intestinal bacterial strains. J Nat Med. 2008;62(4):456-60.

57. Spence RD, Voskuhl RR. Neuroprotective effects of estrogens and androgens in CNS inflammation and neurodegeneration. Front Neuroendocrinol. 2012;33(1):105-15. 


\section{Figures}

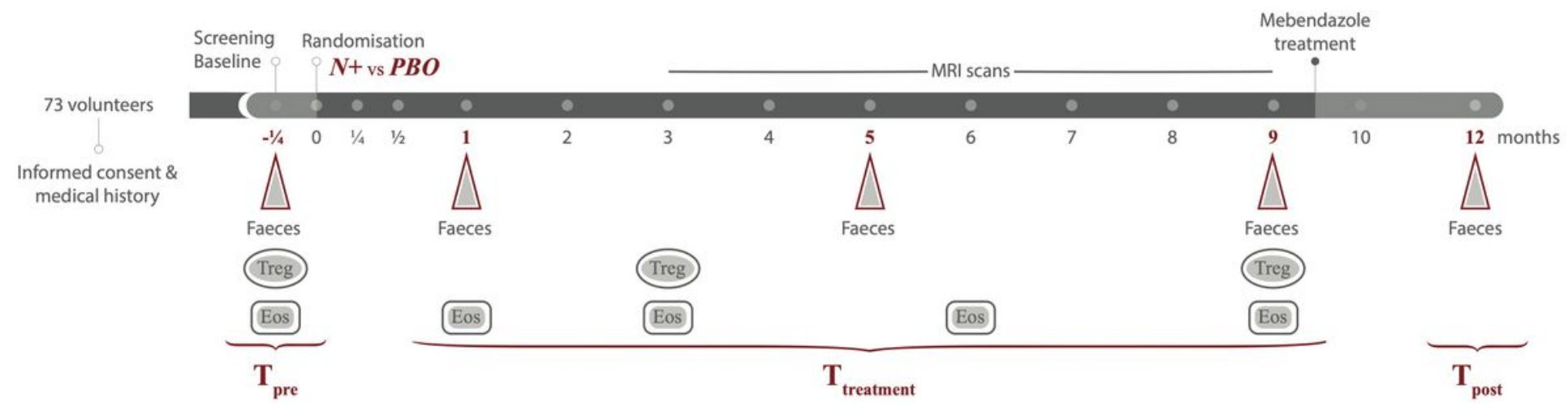

\section{Figure 1}

Overview of the clinical study design. A total of 73 patients suffering from relapsing multiple sclerosis (RMS) were included in the trial and randomly assigned to the two treatment arms, i.e. percutaneous infection with $25 \mathrm{~N}$. americanus larvae (worm), or placebo treatment with pharmacopoeial grade water (PBO). Triangles indicate the timepoints corresponding to faecal sample collection for metagenomic sequencing, whilst ellipses and rectangles indicate collection of blood samples for assessment of regulatory T cell (Treg) and eosinophil (Eos) counts, respectively. Samples collected prior to hookworm infection = Tpre; samples collected at one, five, and nine months post-infection/placebo treatment $=$ Ttreatment; samples collected post-anthelmintic treatment $=$ Tpost. 
a)

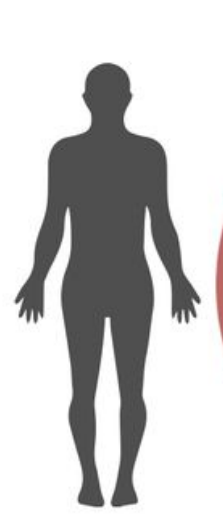

$2.4 \%$

$2.3 \%$

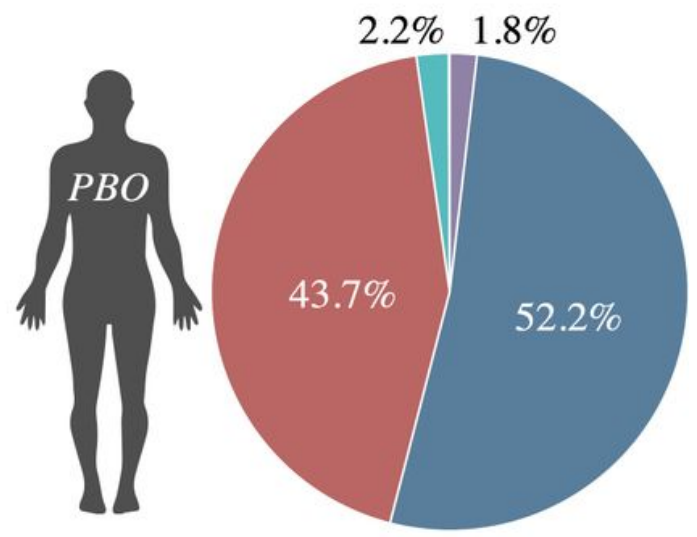

b)

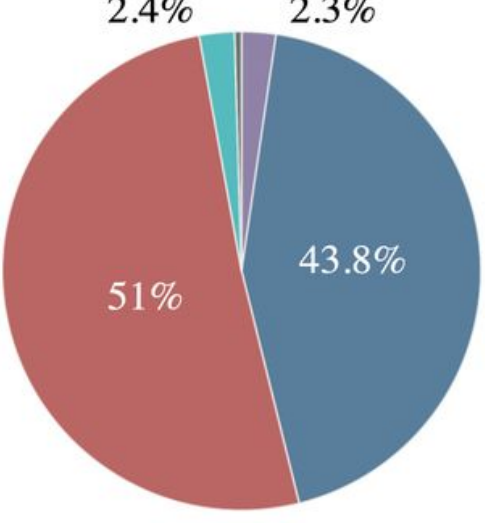

Bacteroidetes

Firmicutes

Actinobacteria

Proteobacteria

Tenericutes

$\mathrm{T}_{\text {pre }}$

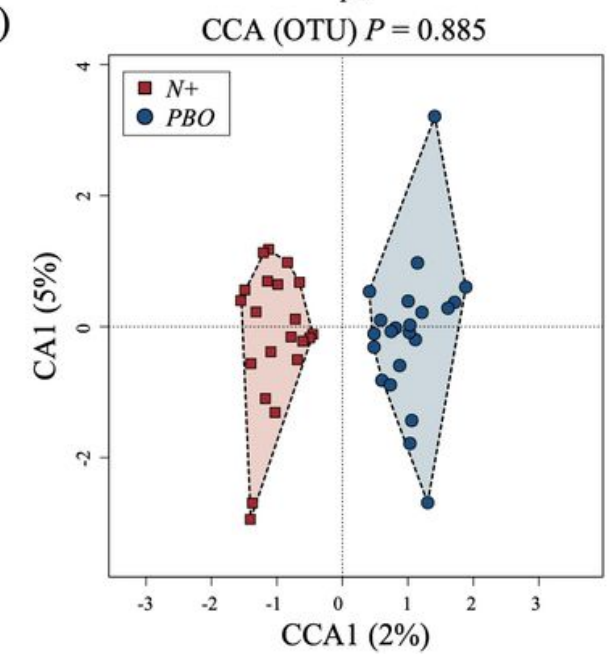

$\mathrm{T}_{\text {treatment }}$

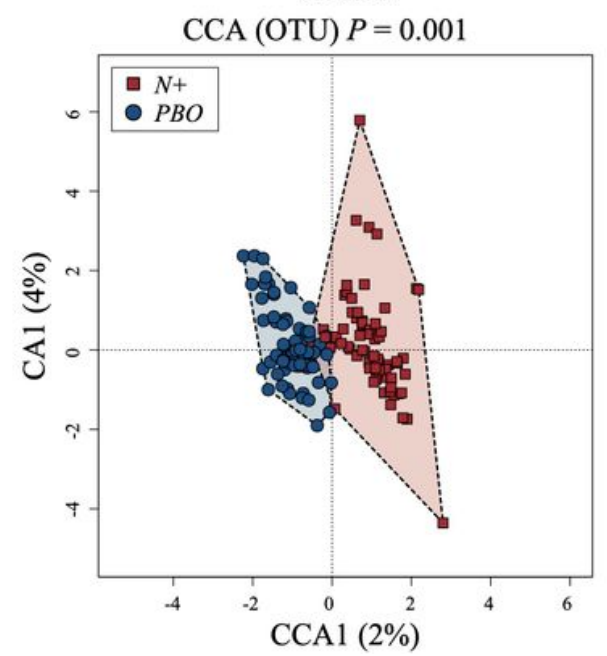

$\mathrm{T}_{\text {post }}$

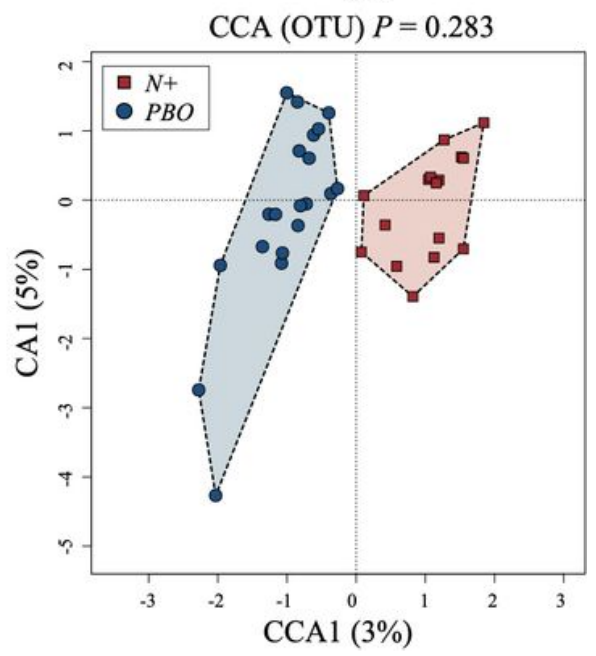

Figure 2

Gut microbial profiles of volunteers with relapsing multiple sclerosis (RMS) infected with Necator americanus $(\mathrm{N}+$ ) or placebo-treated (PBO). (a) Relative abundances of bacterial phyla detected in faecal samples from study subjects. Percentages in individual pie chart sections indicate the relative proportion of the corresponding phylum. (b) Differences between the faecal microbial profiles of $\mathrm{N}+$ and PBO subjects one week prior to infection/placebo treatment (Tpre; left), at one, five, and nine months postinfection/placebo treatment (Ttreatment; centre), and two months post-anthelmintic treatment (Tpost; right) ordinated by supervised Canonical Correspondence Analysis (CCA). 
a)
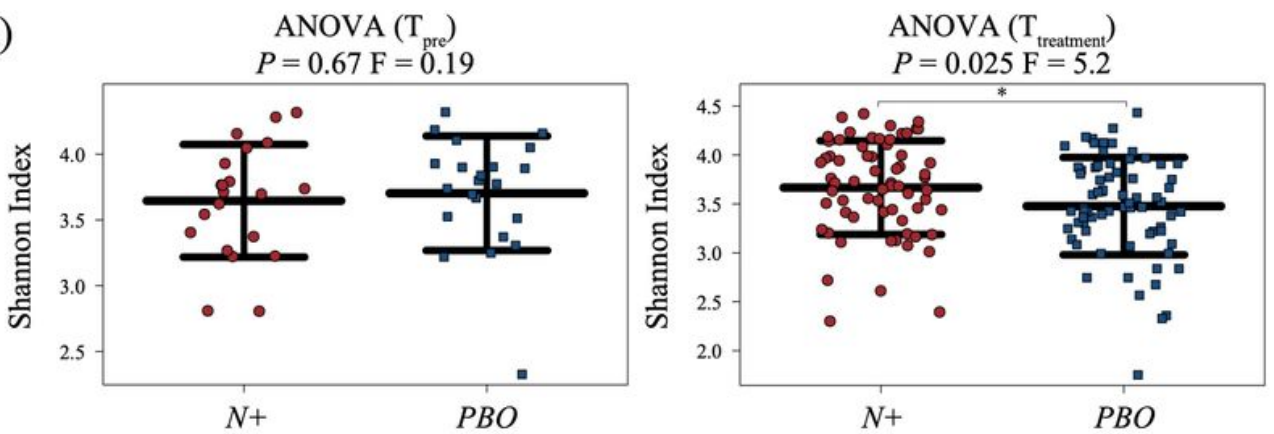

ANOVA $(N+)$

b)

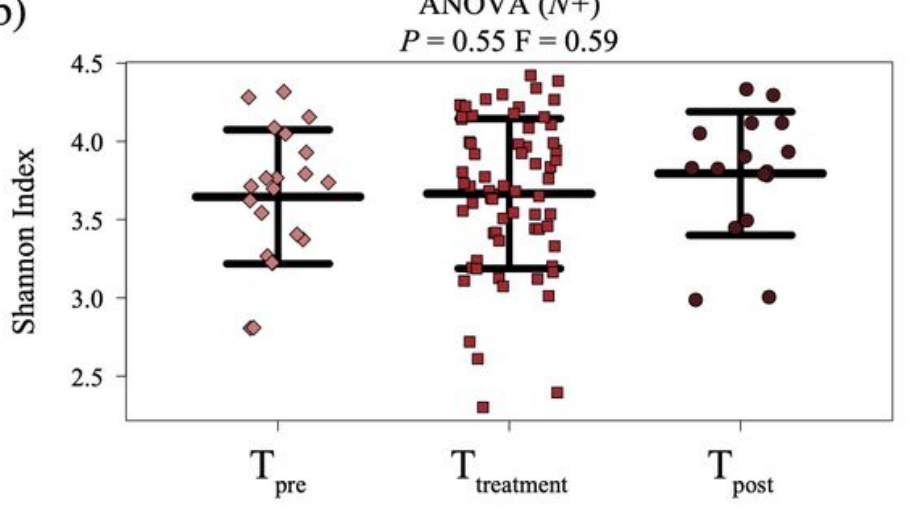

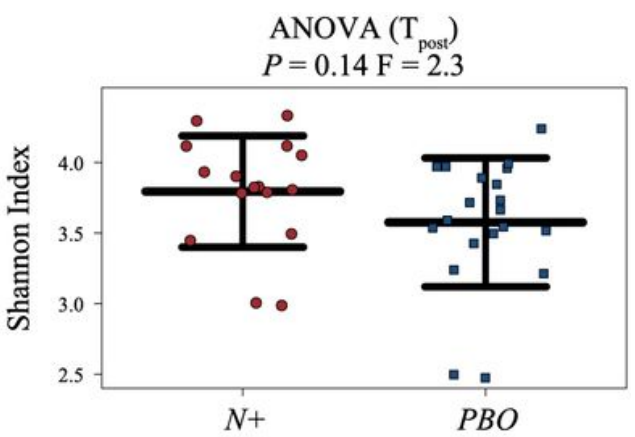

ANOVA (PBO)

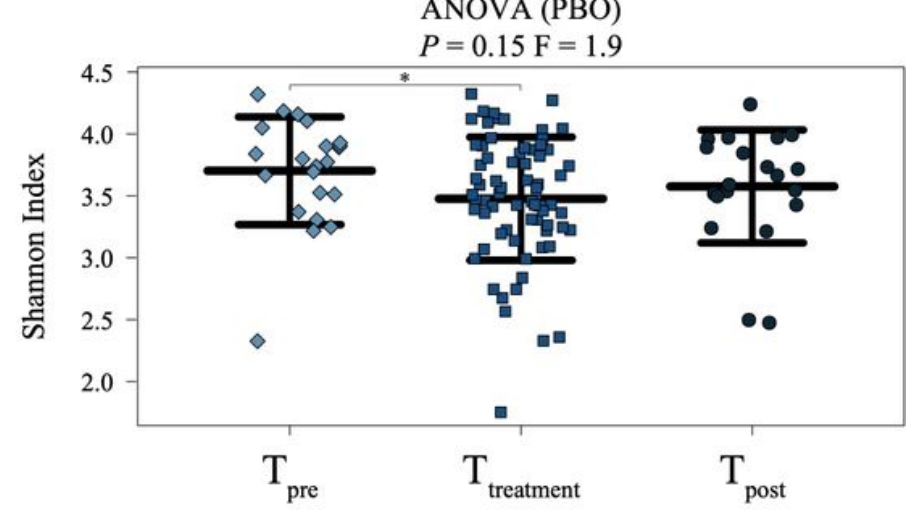

Figure 3

Faecal microbial alpha diversity of volunteers with relapsing multiple sclerosis (RMS) infected with Necator americanus ( $\mathrm{N}+$ ) or placebo-treated (PBO). (a) Differences in faecal microbial Shannon diversity between $\mathrm{N}+$ and PBO subjects one week prior to infection/placebo treatment (Tpre; left), at one, five, and nine months post-infection/placebo treatment (Ttreatment; centre), and two months post-anthelmintic treatment (Tpost; right). (b) Differences in faecal microbial richness between $\mathrm{N}+$ (left) and PBO (right) subjects across all time points. 


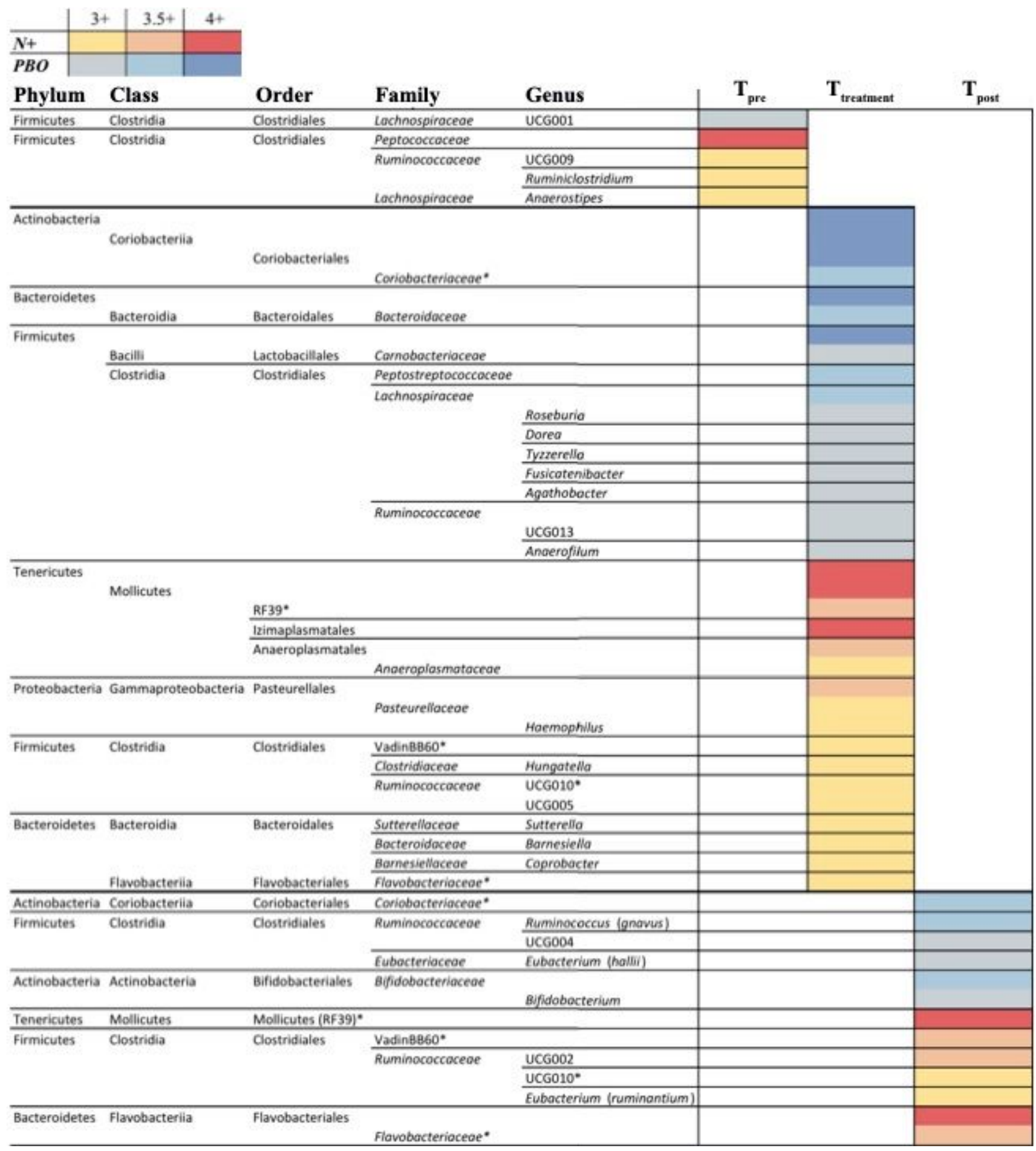

\section{Figure 4}

Differentially abundant bacterial taxa in the faecal microbiota of volunteers with relapsing multiple sclerosis (RMS) infected with Necator americanus $(\mathrm{N}+)$ or placebo-treated (PBO), one week prior to infection/placebo treatment (Tpre), at one, five, and nine months post-infection/placebo treatment (Ttreatment), and two months post-anthelmintic treatment (Tpost) based on Linear discriminant analysis Effect Size (LEfSe) analysis. Colours correspond to Linear Discriminant Analysis (LDA) scores of 4 or higher $(\mathrm{N}+=$ red; $\mathrm{PBO}=$ dark blue), 3.5 to $4(\mathrm{~N}+=$ orange; $\mathrm{PBO}=$ light blue $)$, and 3 to $3.5(\mathrm{~N}+=$ yellow; $\mathrm{PBO}$ $=$ grey $)$. 

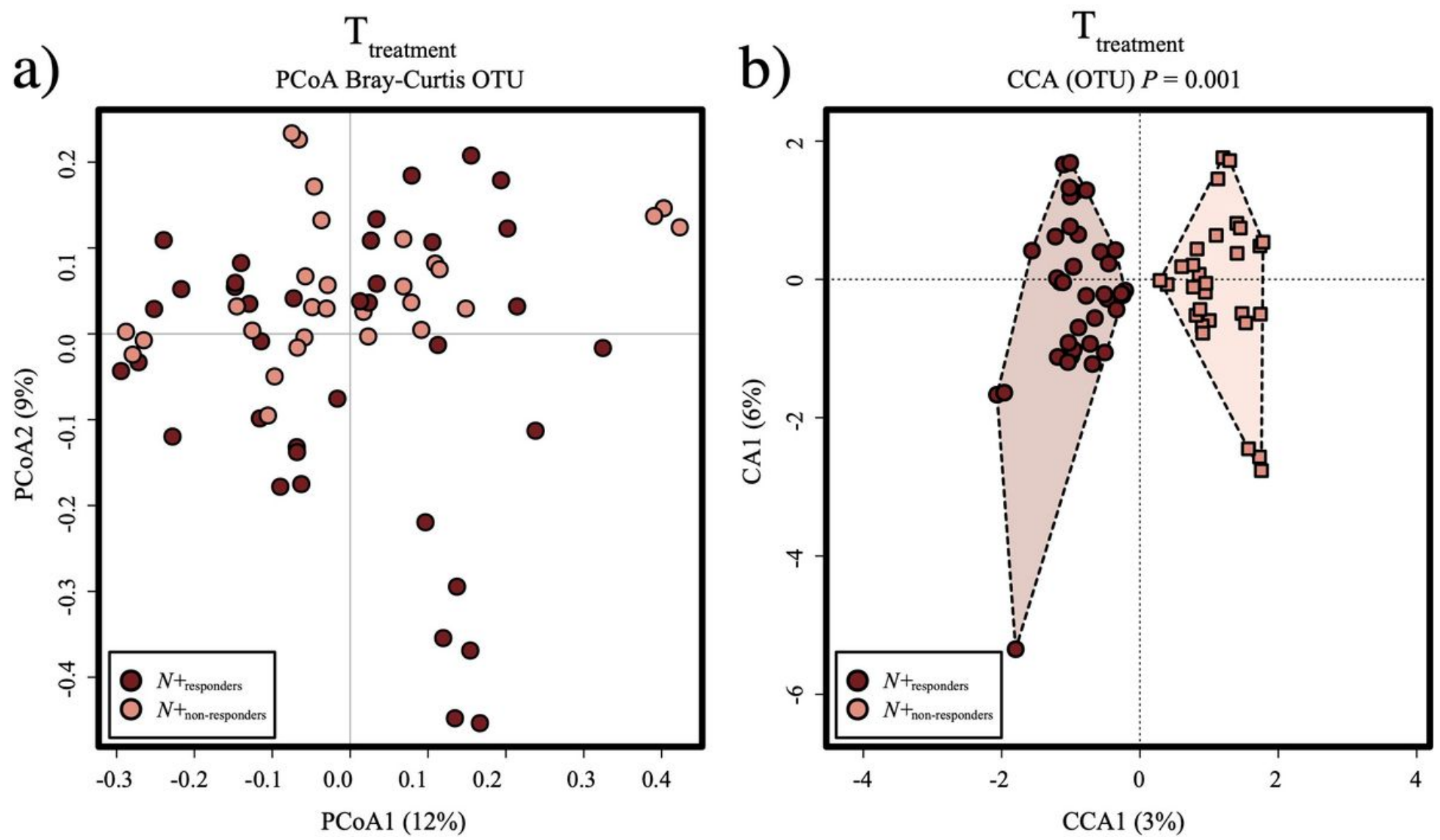

Figure 5

Differences in faecal microbial profiles between Necator americanus volunteers with relapsing multiple sclerosis (RMS) who suffered relapses during the course of the trial ( $\mathrm{N}+$ non-responders) and infected volunteers who did not suffer relapses ( $\mathrm{N}+$ responders), investigated via (a) Principal Coordinates Analysis (PCoA) and (b) Canonical Correspondence Analysis (CCA). 


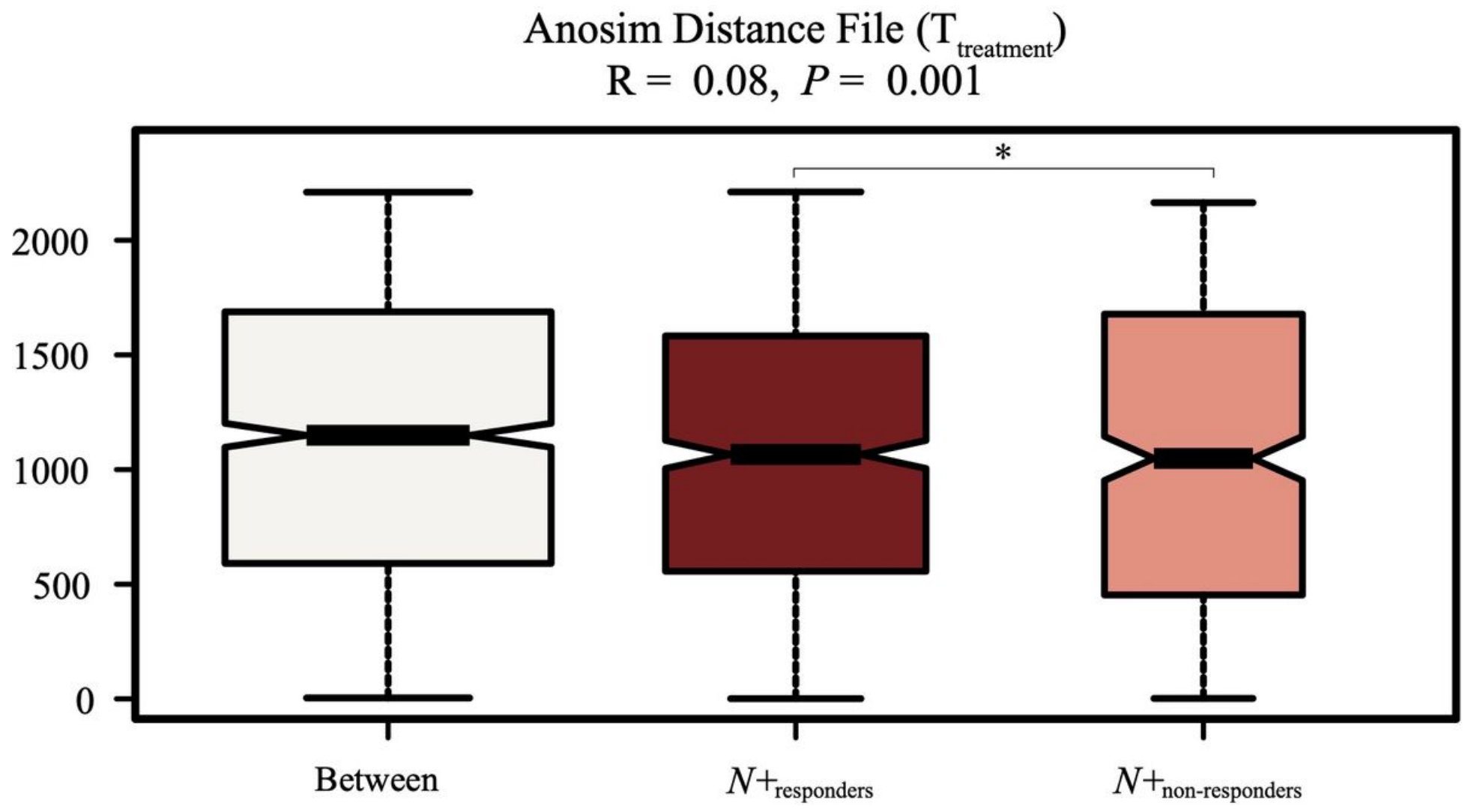

Figure 6

Analysis of similarities (ANOSIM) indicating differences between Necator americanus volunteers with relapsing multiple sclerosis (RMS) who suffered relapses during the course of the trial ( $\mathrm{N}+$ nonresponders) and infected volunteers who did not suffer relapses ( $\mathrm{N}+$ responders). 


\begin{tabular}{|c|c|c|c|c|c|c|c|}
\hline \multicolumn{8}{|l|}{$N t_{\text {regpesitr }}$} \\
\hline $\mathrm{N}+_{\text {ate-rapeede }}$ & & & & & & & \\
\hline Phylum & Class & Order & Family & Genus & $\mathbf{T}_{\text {pre }}$ & $\mathbf{T}_{\text {treatment }}$ & $\mathbf{T}_{\text {post }}$ \\
\hline \multirow[t]{2}{*}{ Bacteroidetes } & \multirow{2}{*}{ Flavobacteriia } & \multirow{2}{*}{ Flavobacteriales } & & & & & \\
\hline & & & Flawobacteriaceae & & & & \\
\hline Bacteroidetes & Bacteroidia & Bacteroidales & Porphyromanodaceare & Parabacteroides & & & \\
\hline Bacteroidetes & Bacteroidia & Bacteroidales & Rikenelliaceoe & Alistipes & & & \\
\hline Firmicutes & Clostridia & Clostridiales & Christensenelliocege & R7 & & & \\
\hline Bacteroidetes & Bacteroidia & Bacteroidales & Prevotelloceae & & & & \\
\hline Firmicutes & Clostridia & Clostridiales & Peptostreptococcaceae & Terrisporobacter & & & \\
\hline \multicolumn{8}{|l|}{ Firmicutes } \\
\hline & \multirow[t]{2}{*}{ Clostridia } & \multirow[t]{2}{*}{ Clostridiales } & Defluvītaleaceoe & & & & \\
\hline & & & & UCG011 & & & \\
\hline & & & Ruminococcaceoe & Ruminiclostridium & & & \\
\hline & & & & UCG004 & & & \\
\hline & & & Christensenelloceae & R7 & & & \\
\hline & & & Eubacteriaceae & Eubocterium (coprostanoligenes) & & & \\
\hline \multirow[t]{3}{*}{ Bacteroidetes } & \multirow[t]{2}{*}{ Flavobacterila } & \multirow[t]{2}{*}{ Flavobacteriales } & & & & & \\
\hline & & & Flavobacteriaceae & & & & \\
\hline & Bacteroidia & Bacteroidales & Porphyromanodaceae & Parabacteroides & & & \\
\hline \multirow[t]{2}{*}{ Tenericutes } & Mollicutes & Anaeroplasmatales & & & & & \\
\hline & & & Anaeroplasmotaceae & & & & \\
\hline Firmicutes & Clostridia & Clostridiales & & & & & \\
\hline & & & Eubacteriaceae & Eubocterium (xylanophilum) & & & \\
\hline & & & & Eubocterium (ruminantium) & & & \\
\hline & & & Ruminococcaceoe & & & & \\
\hline & & & & Anoerofilium & & & \\
\hline & & & & UCG003 & & & \\
\hline & & & & Rominococcus (1) & & & \\
\hline & & & Lachnospiraceoe & Roseburia & & & \\
\hline & & & & Tyzerella (3) & & & \\
\hline & & & & UC6010 & & & \\
\hline & & & & NKAA136 & & & \\
\hline & Negativicutes & Selenomonadaies & & & & & \\
\hline Bacteroidetes & Bacteroidia & Bacteroidales & Prevotelloceae & & & & \\
\hline & & & & Paraprevatella & & & \\
\hline & & & & Prevotella (9) & & & \\
\hline Bacteroidetes & Flavobacteriia & Flavobacteriales & & & & & \\
\hline & & & Flavobacteriaceae & & & & \\
\hline Firmicutes & Clostridia & Clostridiales & Ruminocaccaceoe & Ruminiclostridium & & & \\
\hline Bacteroidetes & Bacteroidia & Bacteroidales & Prevotelloceae & & & & \\
\hline Firmicutes & Clostridia & Clostridiales & Ruminocaccaceae & UCGO03 & & & \\
\hline
\end{tabular}

\section{Figure 7}

Differentially abundant bacterial taxa between Necator americanus volunteers with relapsing multiple sclerosis (RMS) who suffered relapses (N+non-responders) and infected volunteers who did not suffer relapses ( $\mathrm{N}+$ responders), one week prior to infection/placebo treatment (Tpre), at one, five, and nine months post-infection/placebo treatment (Ttreatment), and two months post-anthelmintic treatment (Tpost) based on Linear discriminant analysis Effect Size (LEfSe) analysis. Colours correspond to Linear Discriminant Analysis (LDA) scores of 4 or higher $(\mathrm{N}+$ responders $=$ dark red; $\mathrm{N}+$ non-responders $=$ ochre), 3.5 to $4(\mathrm{~N}+$ responders = red; $\mathrm{N}+$ non-responders $=$ orange $)$, and 3 to $3.5(\mathrm{~N}+$ responders = light red; $\mathrm{N}+\mathrm{non}-$ responders = beige). 
a)

Top 30 predictors $\left(\mathrm{T}_{\mathrm{pre}}\right)$

\begin{tabular}{lccc}
\multicolumn{1}{c}{ Biomarker (genus) } & $P$ & FDR & AUC \\
\hline Parabacteroides & 0.0037 & 0.11 & 0.86 \\
NK4A136 (Lachnospiraceae) & 0.1100 & 0.66 & 0.71 \\
Alistipes & 0.1300 & 0.66 & 0.7 \\
Roseburia & 0.1300 & 0.66 & 0.7 \\
Ruminococcus & 0.1500 & 0.66 & 0.69 \\
R7 (Christensenellaceae) & 0.1700 & 0.66 & 0.68 \\
Dorea & 0.1700 & 0.66 & 0.68 \\
UCG014 (Ruminococcaceae) & 0.1800 & 0.66 & 0.68 \\
Ruminiclostridium & 0.2000 & 0.66 & 0.67 \\
UCG002 (Ruminococcaceae) & 0.3600 & 0.85 & 0.62 \\
Eubacterium (hallii) & 0.4200 & 0.85 & 0.61 \\
Fusicatenibacter & 0.4400 & 0.85 & 0.6 \\
Unclassified & 0.4600 & 0.85 & 0.6 \\
Subdoligranulum & 0.5100 & 0.85 & 0.59 \\
uncultured & 0.5100 & 0.85 & 0.59 \\
Agathobacter & 0.5200 & 0.85 & 0.59 \\
Bacteroides & 0.5700 & 0.85 & 0.58 \\
Anaerostipes & 0.5700 & 0.85 & 0.58 \\
Barnesiella & 0.5700 & 0.85 & 0.58 \\
Blautia & 0.6000 & 0.85 & 0.57 \\
Ruminococcus & 0.6000 & 0.85 & 0.57 \\
UCG005 (Ruminococcaceae) & 0.6500 & 0.85 & 0.56 \\
Oscillibacter & 0.6500 & 0.85 & 0.56 \\
Ruminococcus (torques) & 0.7600 & 0.95 & 0.55 \\
NK4A214 (Ruminococcaceae) & 0.8100 & 0.95 & 0.54 \\
Faecalibacterium & 0.8600 & 0.95 & 0.53 \\
Eubacterium (coprostanoligenes) & 0.9200 & 0.95 & 0.52 \\
Bifidobacterium & 0.9200 & 0.95 & 0.52 \\
Lachnoclostridium & 0.9200 & 0.95 & 0.48 \\
UCG013 (Ruminococcaceae) & 1.0000 & 1.00 & 0.5 \\
& & &
\end{tabular}

b)

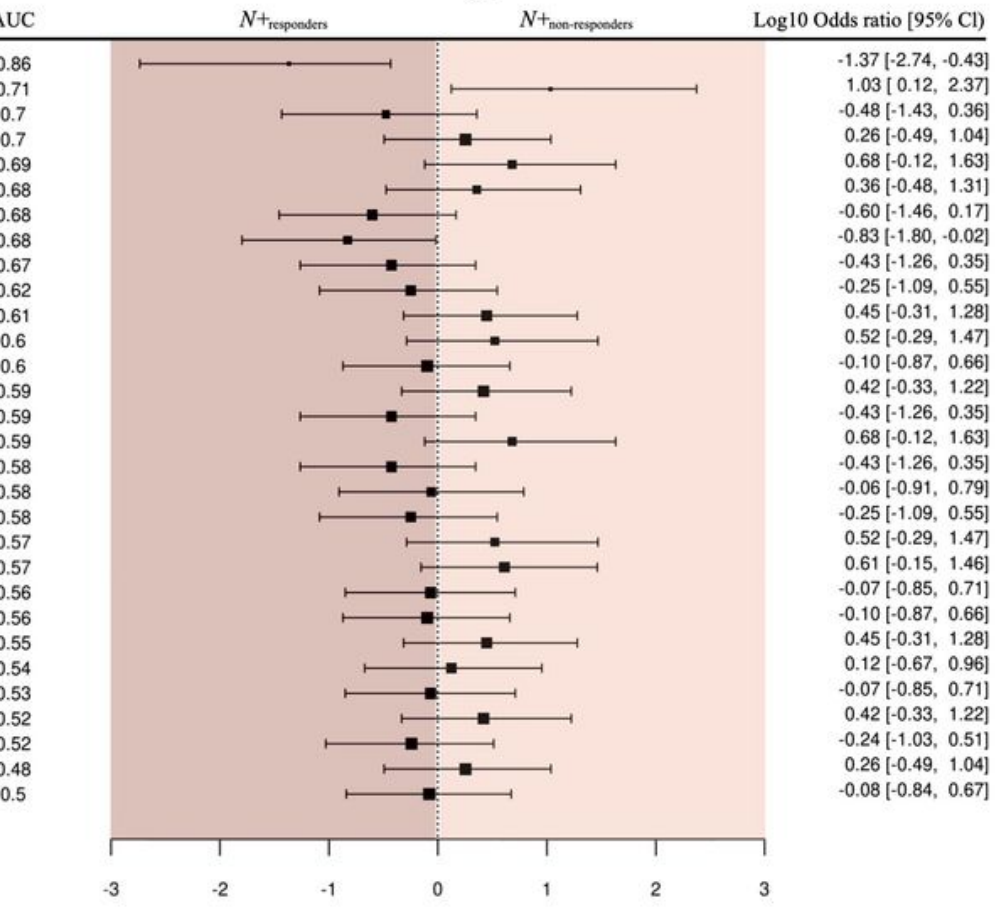

$\log 10$ (OR)

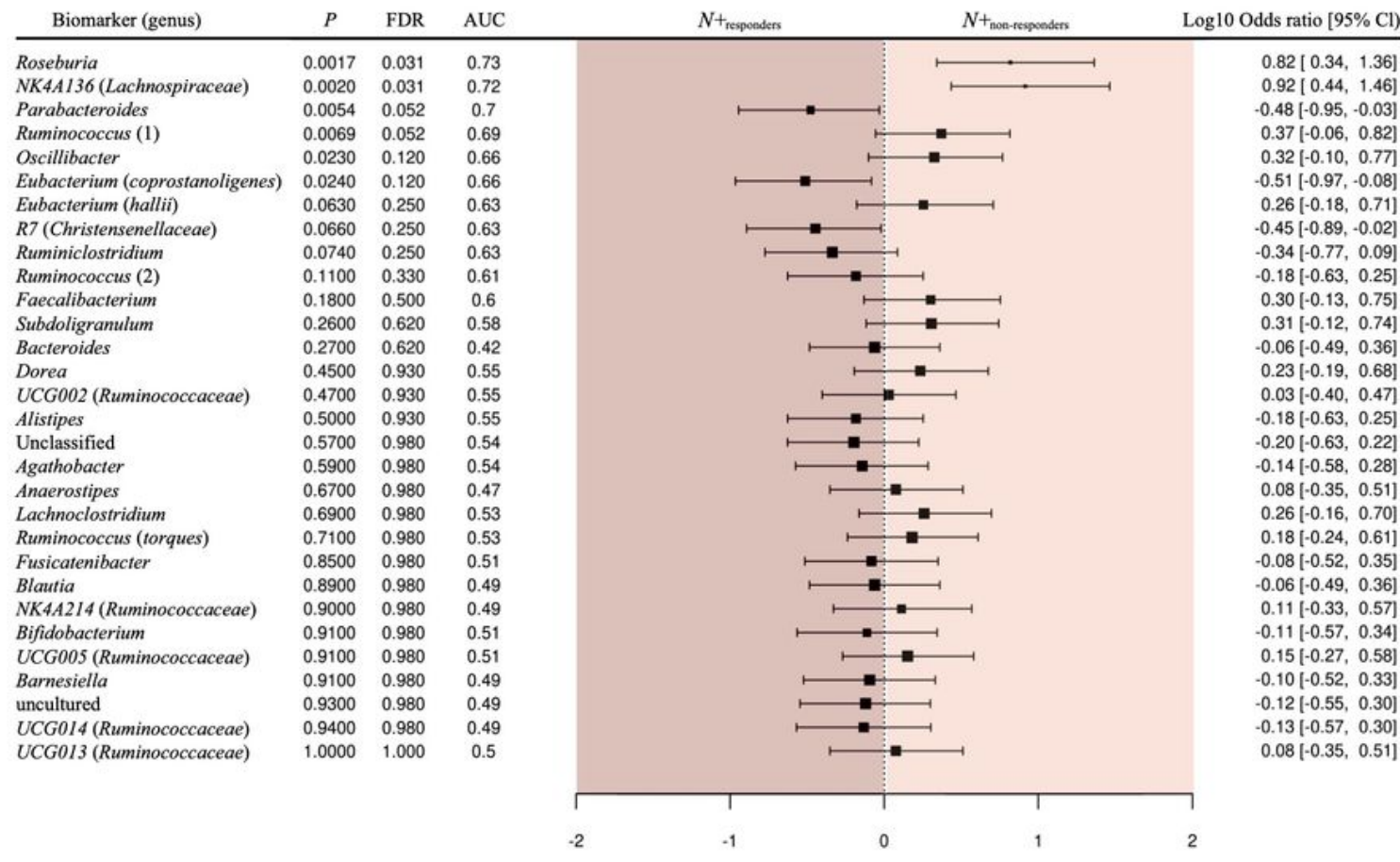

$\log 10$ (OR)

Figure 8

Biomarker analysis listing the 30 faecal bacterial taxa whose relative abundances were associated with positive or negative outcome of hookworm treatment over the course of the WIRMS trial. Biomarker predictions were conducted (a) two weeks prior to the trial (Tpre) and (b) post-infection/placebo treatment (Ttreatment). 


\section{Supplementary Files}

This is a list of supplementary files associated with this preprint. Click to download.

- FigureS2.jpg

- FigureS11.jpg

- FigureS10.jpg

- FigureS7.jpg

- FigureS9.jpg

- FigureS5.jpg

- FigureS6.jpg

- FigureS3.jpg

- FigureS8.jpg

- FigureS1.jpg

- FigureS4.jpg 